\title{
Assessment of Serum Copper Level among Sudanese Patients with vitiligo
}

\section{Mehad Muawia', Suad H H², Gad Allah Modawe ${ }^{3}$}

Corresponding Author: gadobio77@hotmail.com

Received 02 January 2020 Accepted 17 March 2020 Published 31 March 2020

Production and Hosting by Knowledge E

(C) Gad Allah Modawe. This article is distributed under the terms of the Creative Commons Attribution License, which permits unrestricted use and redistribution provided that the original author and source are credited.

Editor-in-Chief:

Prof. Mohammad A. M. Ibnouf

1. Department of Clinical chemistry, Faculty of Medical Laboratory Sciences, Al Neelain University, Omdurman, Sudan

2. Department of Dermatology, Faculty of Medicine and Health Sciences, Omdurman Islamic University, Omdurman, Sudan

3. Department of Biochemistry, Faculty of Medicine and Health Sciences, Omdurman Islamic University, Omdurman, Sudan

\section{Abstract}

Background: Vitiligo is a common skin disease of unknown etiology characterized clinically by depigmented patches, which can be localized or generalized; it usually runs a chronic course with an un predictable outcome and failure of complete cure in many affected individuals. Many communities consider it a contagious disease which leads to a great psychological and social stigma for patients; previous studies showed that copper might be associated with the pathogenesis of vitiligo. The aim of this study was to assess copper level in Sudanese vitiligo patients.

Methods: This is a case-control study conducted in dermatology clinics in Khartoum state during the period from November 2018 to February 2019. Blood samples were obtained from 100 participants, 50 from vitiligo patients and 50 from non-vitiligo subjects representing a control group. Serum copper was measured by mind-ray (automation). Results: A highly significant increase $(p=0.000)$ in the copper level was seen in vitiligo patients compared with the control group. Of the total number of patients, 17 (34\%) were females and 33 (66\%) were males. According to the duration of the disease, the copper level was significantly increased in patient group with a disease duration of > one year compared to the patient group six months - one year and patient group < 6 months; we found no significance of the family history, $18 \%$ of the case group had a family history while 42 (82\%) had no family history.

Discussion: The relationship between the serum level of copper and vitiligo has been assessed by many studies. Copper is one of the trace elements that was found to be important for tyrosinase enzyme that catalyzes the first steps in melanin synthesis in the skin. Some studies showed that the disease was associated with low serum levels of copper and since vitiligo is a disease that is characterized clinically by white areas of skin with no melanin, these studies seem to be logical. However, in this study, the serum level of copper was found to be high in vitiligo patients which might be justified by the release of copper from the destroyed melanocytes. Another justification might be a defect in the carrier protein for copper.

Conclusion: The study found that the serum copper was significantly increased in vitiligo patients compared to the control and it is recommended that serum copper level and copper profile should be assessed routinely in vitiligo patients. 
Key words: vitiligo; depigmentation; copper, Sudanese

\section{Introduction}

Vitiligo is a chronic idiopathic skin disease, characterized by sharply marginated depigmented patches [1]. Lesions often start on sun-exposed areas [2]. Both genetic and environmental factors are believed to play a role in the pathogenesis of the disease $[1,2]$. The distribution of the disease can be unilateral segmental or non-segmental [1]. Vitiligo is estimated to account for $1 \%$ of the general population globally [3], however, in some populations, the incidence has reached 2-3\% [4]. Patients who are stigmatized for the disease can experience depression and other psychological mood disorders [5]. The risk factors for developing vitiligo include family history and other autoimmune diseases [2]. The disease is usually diagnosed clinically. The diagnosis is confirmed by tissue biopsy and histopathological study [2]. Vitiligo should be differentiated from other skin conditions in which there is decrease in or loss of pigment, for example, tinea versicolor, piebaldism, idiopathic guttate hypomelanosis, etc. [6]. There is no cure for vitiligo [1], but it can be treated with the many available options, including topical steroids, sunscreens, phototherapy, etc. [1, 2]. Melanin is the pigment majorly responsible for skin color; it is synthesized from amino acid tyrosine in the melanosomes that are found within epidermal melanocytes. Tyrosinase enzyme catalyzes the first two reactions in the pathway of melanogenesis [7]. It is found in animal and plant tissues responsible for the production of melanin as well as other pigments in these tissues [8]. Melanin is an effective absorbent of ultraviolet rays (UV) [10] and prevents the skin from its harmful effects. Some studies have shown that there is a reduced incidence of some types of skin cancers in individuals with dark skin compared to others, but the relationship between photo protection and the incidence of skin cancer is not clearly understood [11]. Tyrosinase is a copper-containing enzyme encoded by the TYR gene in humans [9].

Copper is a trace element needed in small amount in the diet, the proximal small intestine is recognized as the main site of dietary copper absorption in mammals. The transport of copper from the intestinal lumen into the intestinal mucosa is a carriermediated process involving a saturable transport component. The overall intestinal copper uptake is influenced by amino acids, ascorbic acid, and other dietary factors [12]. Once in mucosal cells, approximately $80 \%$ of the newly absorbed copper is in the cytosol, mainly bound to metallothionein (MTs). These are low-molecular weight-inducible proteins with many functions including homeostasis, storage, transport, and detoxification of metals. The MTs bind to many metals, but in normal circumstances only $\mathrm{Zn}, \mathrm{Cu}$, and $\mathrm{Cd}$ binding is 
significant [13]. After passing through the enterocytes, copper enters the portal circulation where it is bound to carrier proteins (primarily albumin), peptides, and amino acids and is transported to the liver, with lesser amounts entering the kidneys.

Recently, many studies have been conducted to show that copper plays an important role in pigmentation, it accelerates the oxidation of dopa by skin extracts containing dopa-oxidase. Furthermore, the action of copper in pigmentation was not clear until Gorter [14], in 1935, conclusively demonstrated the fact that copper-free diets resulted in depigmentation of the hair of rats, rabbits, and cats and that this depigmentation disappeared following the administration of copper. On the other hand, no effect on melanogenesis was obtained if other minerals or vitamins were added to the diet [14].

In an old study submitted in Philadelphia 1931, Cunningham [15] made an observation relevant to this problem; he noted that the skin of black-coated animals contained more copper than the white-coated ones and that copper was concentrated mainly in the epidermis and "in vitro" experiments demonstrated the fact that copper accelerated the oxidation of dopa by skin extracts containing dopa-oxidase [15]. In the same year, Sarata [15] made the first definite attempt to correlate the copper content of skin with its degree of pigmentation. A study carried out in mottled dogs and cats showed that the copper content of pigmented hair was much higher than that of the nonpigmented hair of the same animal. Moreover, generally speaking, copper was found to be present in greater amounts in a skin covered by dark hair than the skin underlying the colorless hairs [15].

In 1934, Schroeder, Gruenberg, and Schade, quoted by Cornbleet [16], demonstrated that vitamin "C" inhibits the dopa reaction. Cornbleet [16] found that pigmentary precipitates can occur in a solution of dopa under the action of UV light alone, Buthe was able to accelerate this reaction considerably by adding copper to the solution. On the other hand, the addition of vitamin $C$ tended to slow down the reaction or, in other words, to neutralize the catalytic effect of copper. Cornbleet concluded that the presence of these two substances, having antagonistic actions, so far as the oxidation rate of dopa is concerned, makes pigment formation susceptible to ready physiologic control [16].

Also, a study conducted at the Faculty of Medicine, Fayoum University showed that serum Zn levels were lower in different studied groups but it was much lower in the vitiligo group and that the serum $\mathrm{Cu}$ levels in the vitiligo group were insignificantly higher compared to the control group. Hence, serum $\mathrm{Zn}$ and $\mathrm{Cu}$ may have an effect on the vitiligo disease as $\mathrm{Zn}$ in combination with other micronutrients such as $\mathrm{Cu}$, cobalt, nickel, iron, manganese, and $\mathrm{Ca}++$ plays an important role in the process of melanogenesis [17]. 
In another study conducted at the Central South University, Changsha, China in 2014, it was noted that that out of the 16 studies that detected serum Cu level, 6 reported no statistically significant Cu level change in both the groups while 10 presented a significant decrease in the Cu levels in the vitiligo group. Thereafter, a random effect model was used for meta-analysis. In the pooled analysis, there was a significant decrease in the Cu levels in the vitiligo group [16].

Copper is essential for human heath, it is crucial for growth and brain development and helps fight dangerous infections, according to some reports in the literature review, lower copper increases the susceptibility to vitiligo, vitiligo is one of the most common skin diseases, it can persist for years and result in disfigurement and permanent scarring, it can cause serious adverse effects on psychological development resulting in emotional problems like withdrawing from society and depression. However, most of the researches were directed to determine the pathological effect due to changes in the level of copper, since there is a strong relationship between serum copper level and vitiligo, hence this study was designed to highlight the relation between serum copper level and Vitiligo in Sudanese patients. The aim of this study was to assess the serum copper level in patients with vitiligo.

\section{Materials and Methods}

This study is a case-control hospital-based study carried out at Sudanese dermatology clinics and the Khartoum Teaching Hospital for Dermatology, Khartoum city, Khartoum state. The study was conducted during November 2018 to January 2019. The study population was 100, with 50 vitiligo patients and 50 non-vertigo patients representing the control group.

The study populations were matched in age and sex, the age ranged between 16 and 60 years, the number of males studied was 33 (66\%) and females 17 (34\%).

Sudanese patients with any type of vitiligo were included in the study. Whereas, all subjects with leukoderma secondary to other causes, subjects with a history of other obvious skin diseases, patients undergoing treatment with copper or any history of copper intake for six weeks before this study, and subjects who suffered from any other systemic diseases such as hepatic cirrhosis, viral hepatitis, neoplastic condition, myocardial infarction, steatorrhea, renal failure, pregnancy, consumption of oral contraceptive pills, and GIT troubles (like dyspepsia, diarrhea, etc.) were excluded from the study. 
A blood sample of $3 \mathrm{ml}$ was collected in plain containers from each volunteer under optimum condition. The blood was centrifuged at 5000 r.p.m for 10 min which was stored in small aliquots and kept in a deep freezer $\left(-20^{\circ} \mathrm{C}\right)$ until analyzed. Data were collected from patients using questionnaire forms. After explaining the aim of the study, verbal consent was taken from all participants before sample collection.

Serum copper level was analyzed through spectrophotometric method using Mindray. The precision and accuracy of the methods used in this study were checked each time using a control material.

This study was approved by the committee of department of clinical chemistry, Faculty of Medical Laboratory Sciences, Al-Neelain University, Khartoum, Sudan.

The collected data were analyzed using the statistical package of social sciences (SPSS) version 21.0. The results were expressed using (Mean \pm SD) figures and tables.

\section{Results}

The study enrolled 50 vitiligo patients and 50 healthy subjects as a control group with matched age and sex. It was conducted at different dermatology clinics in Khartoum city, Khartoum state. The aim was to assess the serum copper level and to compare the results of the case and control groups of the study populations.

Figure 1 shows the distribution of patients according to gender. The number of males among the vitiligo patients was 33 (66\%) and females 17 (34\%).

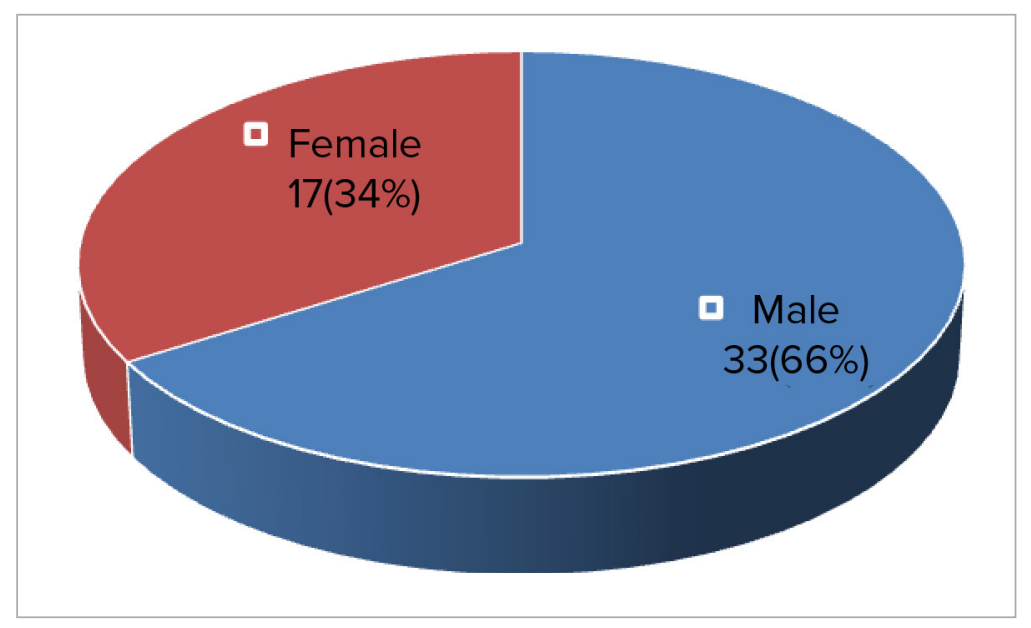

Figure 1: Distribution of Patients According to the Gender. 
Figure 2 shows the distribution of patients according to family history. Nine vitiligo patients (18\%) had a family history of the disease while $41(82 \%)$ had no family history.

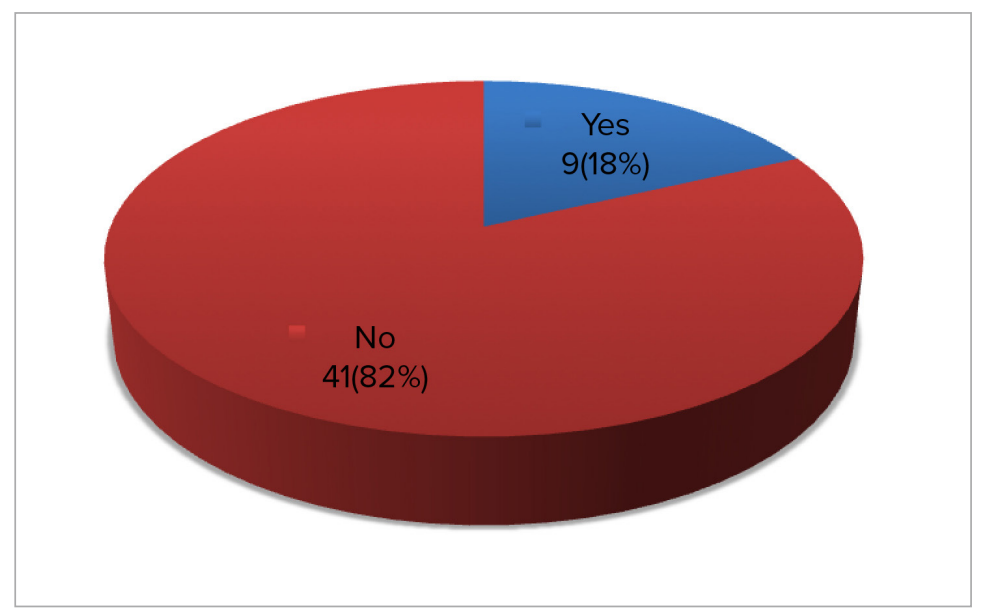

Figure 2: Distribution of Patients According to the Family History of vitiligo Disease.

Figure 3 shows the distribution of patients according to the duration of the disease. The patient group (6 months-1 year) had the longest duration of the disease, 27 (54\%) followed by the group with $>1$ year duration comprising 15 (30\%) and the group with $<6$ months duration comprising 8 (16\%).

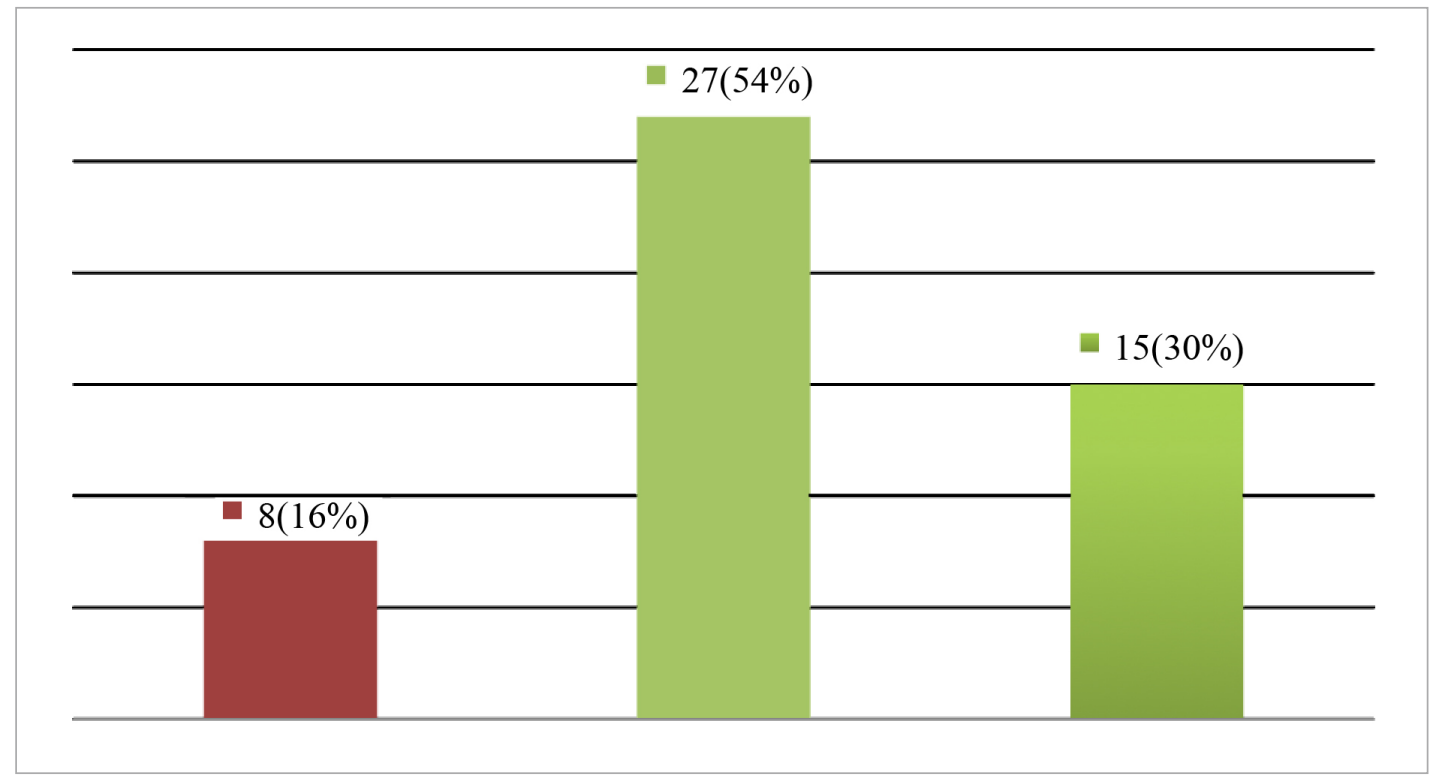

Figure 3: Distribution of Patients According to the Duration of vitiligo Disease.

Table 1 shows a mean comparison of age in the study populations, the age was matched between the case and control groups, the age ranged between 16 and 60 years. 
TABLE 1: Mean Comparison of Study Parameter Across Age.

\begin{tabular}{|l|c|c|c|c|}
\hline Variable & Group & Min. & Max. & Mean \pm SD \\
\hline Age (Years) & Case & 16.00 & 60.00 & $32.52 \pm 11.86$ \\
\hline & Control & 16.00 & 60.00 & $32.72 \pm 10.31$ \\
\hline
\end{tabular}

Table 2 shows the mean comparison of study parameter in case versus control group. The copper level in the case and control groups was $21.69 \pm 5.17 \mathrm{~m} \mu \mathrm{ol} / \mathrm{l}$ and $18.05 \pm 3.51 \mathrm{~m} \mu \mathrm{ol} / \mathrm{l}$, respectively). The serum copper was significantly increased in vitiligo patients compared to the control group ( $p$ value $=0.000$ )

TABLE 2: Mean Comparison of Study Parameters in Case versus Control Groups.

\begin{tabular}{|l|c|c|c|}
\hline Parameter & Group & Mean \pm SD & $p$ value \\
\hline Copper level $\mathbf{m \mu o l} / \mathbf{I}$ & Case & $21.69 \pm 5.17$ & 0.000 \\
\hline
\end{tabular}

Table 3 shows mean comparison of study parameter across gender. There is no difference between males and females.

TABLE 3: Mean Comparison of Study Parameter across Gender.

\begin{tabular}{|l|c|c|c|}
\hline Parameter & Gender & Mean \pm SD & $p$ value \\
\hline Copper level $\mathrm{m} \mu \mathrm{ol} / \mathrm{I}$ & Male & $22.18 \pm 5.91$ & 0.274 \\
\hline
\end{tabular}

Table 4 shows mean comparison of study parameter across family history. The copper levels in family history were $22.48 \pm 5.53 \mathrm{m \mu ol} / \mathrm{l}$ and $22.48 \pm 5.53 \mathrm{m \mu ol} / \mathrm{l}$, respectively, there were insignificant differences with $p$ value $=0.612$.

TABLE 4: Mean Comparison of Study Parameter across Family History.

\begin{tabular}{|l|c|c|c|}
\hline Parameter & Family History & Mean \pm SD & $p$ value \\
\hline Copper level muol/I & Yes & $22.48 \pm 5.53$ & 0.612 \\
\hline
\end{tabular}

Table 5 shows the mean comparison of study parameter across the duration of vitiligo disease. There was no significant difference of serum copper level regarding the duration of the disease.

TABLE 5: Mean Comparison of Study Parameter across the Duration of Disease.

\begin{tabular}{|l|c|c|}
\hline Duration & Mean \pm SD & $p$ value \\
\hline$<6$ Months & $20.66 \pm 4.05$ & \\
\hline 6Months-1 Year & $20.91 \pm 4.52$ & 0.905 \\
\hline$>1$ Year & $23.63 \pm 6.47$ & 0.192 \\
\hline
\end{tabular}


Figure 4 shows the correlations between the age and the serum copper level. There was a negative correlation between age and serum copper level $(r=0.230, p=0.108)$.

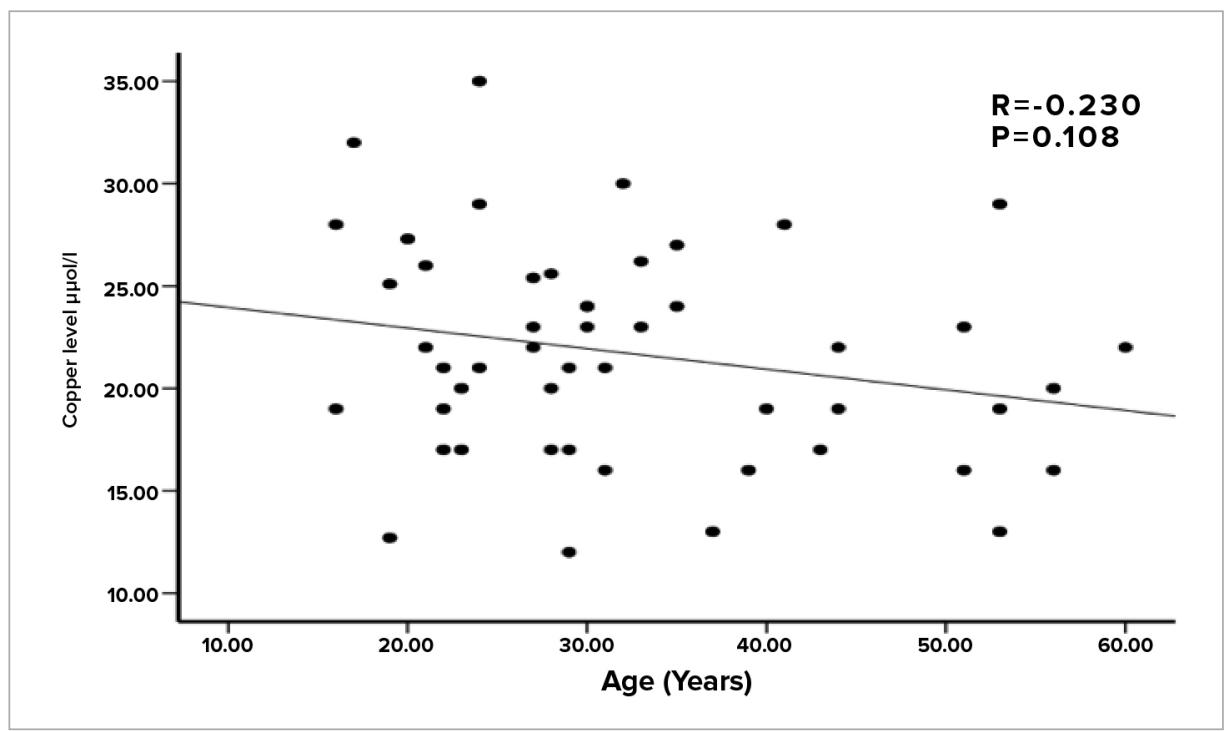

Figure 4: The Correlations Between Age and Serum Copper Level in the Study Group.

\section{Discussion}

Vitiligo is a chronic skin disease that is considered as a stigma to patients in certain communities, it is wrongly believed to be contagious. The etiology of vitiligo is still not known and the effect of all therapies till now doesn't seem promising. Many studies have been conducted on vitiligo pathogenesis but it is still idiopathic and no result was conclusive. Some studies have demonstrated the role of trace elements such as copper, zinc, iron, etc. in melanogenesis with different results and conclusions. The role of copper in melanogenesis has been studied since 1931 when Keil and Nelson had observed that the color of the hair of experimental rats had turned white when they were fed with only milk, which is a copper-deficient diet while being studied for milk anemia [1]. Cunningham in the same year found that the white-coated animals have lower copper levels in their skin than the black-coated ones, he also found that copper is concentrated mainly in the epidermis [2]. This observation is very important. The first observation pointed to the possible role of copper in melanin synthesis and the second is more supportive to this hypothesis since melanogenesis occurs in the epidermis, but of course till that time no one had talked about the nature of melanocytes and their location within epidermal keratinocytes. Since melanogenesis takes place in the skin of animals and humans, researchers started to study the role of copper and other 
trace elements in animals. These studies had raised the suspicion of the role of copper in the pathogenesis of vitiligo in humans and since that time many other researches had talked about the possible role of trace elements in the etiology of vitiligo with the hope of finding promising treatment for this condition which is still idiopathic and all the possible etiologies are stated as hypotheses. The results of this study pointed to slightly increased incidence of vitiligo in male $22.18 \pm 5.91$ versus female $20.74 \pm$ 3.27 with a $P$-value of 0.274 , in agreement with Wang et al. [18] who found a higher prevalence of the disease in male than in female. The male to female ratio was 1.6:1, which is similar to that reported by McBurney [19] but in disagreement with Lu et al. [20] who reported that vitiligo is distributed equally in men and women. The present study was conducted on the age group ranging from 16-60 years with a mean age of 31 years, similar to Marwa Salem et al.'s study [21], in which she included 50 patients with vitiligo of both sexes: 33 females (60\%) and 17 males (34\%). Fifty volunteers were included as a control group. The age in vitiligo group ranged between 15 to 60 years with a mean \pm SD of $36.74 \pm 14$ years and in control group ranged from 15 to 60 years with a mean \pm SD of $31.28 \pm 9.49$ years, and disagreed with a study which reported that the mean age of onset was 18.9 years, while another study found that the mean age of onset was 23.7 years old. The present findings observed that there is a high concentration of copper in vitiligo patients as compared to healthy controls, agreed with Helmy et al. [22], who showed that Cu levels were significantly higher in active vitiligo patients compared to the control. On the contrary, Wasan and Al-Rubayee [23], Wu et al. [24], Kang et al. [27], Wang et al. [18], Shi et al. [25], and Li et al. [26] reported no statistically significant Cu level change between the vitiligo patients and the control group and all of them agreed with Marwa Salem et al. (26), who also reported that serum Cu was insignificantly higher in vitiligo group compared to the control group. Of the 16 studies that detected the serum Cu level, six studies [24, 25, 28, 32, 37] reported no statistically significant $\mathrm{Cu}$ level change between the vitiligo and the healthy groups. The others presented significant decrease of Cu level in the vitiligo group (18). Melanin's are colloidal pigments and have a high affinity for metal ions; therefore, $\mathrm{Cu}$ is found in high levels in pigmented tissues involved in melanin synthesis. As melanocytes degenerate in vitiligo patients, less $\mathrm{Cu}$ is utilized for the melanin synthesis, which consequently raise levels of $\mathrm{Cu}$ in serum in vitiligo patients (18). According to the results of the study we reported that the disease has no association with family history, in the mean comparison of study parameter across family history. The copper levels in family history were $(22.48 \pm 5.53 \mu \mu \mathrm{ol} / \mathrm{l}$ and $22.48 \pm 5.53 \mu \mu \mathrm{ol} / \mathrm{l}$, respectively). The $p$ value $=0.612$ 
showed no significant differences, disagreeing with a Chinese study that reported $20 \%$ of the patients with positive family history in first-degree relatives [18].

\section{Conclusions}

This study concluded that serum copper was highly and significantly increased in Sudanese vitiligo patients studied compared to the control group.

\section{Recommendation}

In our study, we recommend encouragement of healthy diets containing copper and all other trace elements important for enzymatic actions to prevent serious diseases, for example, vitiligo, measurement of copper profile, routine investigations for copper, and other trace elements for vitiligo patients, and further studies regarding the role of other trace elements need to be performed in vitiligo patients because the etiopathogenesis of the disease is still unclear and also the treatment of vitiligo still represents a great challenge.

\section{References}

[1] Ezzedine K, Eleftheriadou V, Whitton M et al. (2015). Vitiligo. Lancet. 386(9988): 74-84.

[2] NIAMS (2014). Archived from the original on 21 August 2016. Retrieved 11 August 2016.

[3] Whitton M, Pinart M, Batchelor JM et al. (2016). Evidence-based management of vitiligo: summary of a cochrane systematic review. The British Journal of Dermatology. 174(5): 962-969.

[4] Krüger C, Schallreuter KU (2012). A review of the worldwide prevalence of vitiligo in children/adolescents and adults. International Journal of Dermatology. 51(10): 1206-1212.

[5] Picardi A, Pasquini P, Cattaruzza MS et al. (2003). Stressful life events, social support, attachment security and alexithymia in vitiligo. A case-control study. Psychotherapy and Psychosomatics. 72(3): 150-158.

[6] Birlea SA, Spritz RA, Norris DA (2007). Vitiligo. In L. A. Goldsmith, S. I. Katz, B. A. Gilchrest, A. S. Paller, D. J. Leffell, and K. Wolff (Eds.), Fitzpatrick's Dermatology in General Medicine (7th ed.). New York: McGraw-Hill Professional. 
[7] Kumar CM, Sathisha UV, Dharmesh S et al. (2011). Interaction of sesamol (3,4-methylenedioxyphenol) with tyrosinase and its effect on melanin synthesis. Biochimie. 93(3): 562-569.

[8] Stevens LH, Davelaar E, Kolb RM et al. (1998). Tyrosine and cysteine are substrates for blackspot synthesis in potato. Phytochemistry. 49(3): 703-707.

[9] Barton DE, Kwon BS, Francke U (1988). Human tyrosinase gene, mapped to chromosome 11 (q14----q21), defines second region of homology with mouse chromosome 7. Genomics. 3(1): 17-24.

[10] Meredith P, Riesz J (2004). Radiative relaxation quantum yields for synthetic eumelanin. Photochemistry and Photobiology. 79(2): 211-216.

[11] Brenner M, Hearing VJ (2008)

[12] Fuentealba IC, Aburto EM (2003). Animal models of copper-associated liver disease. Comparative Hepatology. 2(1): 5.

[13] Ligoxygakis P (2001). Copper transport meets development. Trends in Genetics. 17(8): 442.

[14] Becker SW (1933). Arch Derm Syph. 28: 497.

[15] Knin HL, Nelson VH (1931). Journal of Biological Chemistry. 93: 49; Cunningham IJ (1931). Some biochemical and physiological aspects of copper in animal nutrition. Biochemical Journal. 25(4): 1267-1294.

[16] Mabson P (1935). Bull. soc. franc. de dermat. et syph. (Reunion dermat., Stras-bourg). 42: 1112.

[17] Pillsbury DM, Kulchar GV (1933). Arch Derm Syph. 27: 36.

[18] Wang X, Du J, Wang T et al. (2013). Prevalence and clinical profile of vitiligo in China: a community-based study in six cities. Acta Dermato-Venereologica. 93(1): 62-65.

[19] McBurney El (1979). Vitiligo. Clinical picture and pathogenesis. Archives of Internal Medicine. 139(11): 1295-1297.

[20] Lu T, Gao T, Wang A et al. (2007). Vitiligo prevalence study in Shaanxi Province, China. International Journal of Dermatology. 46(1): 47-51.

[21] (2018). The Egyptian Journal of Hospital Medicine. 70(2).

[22] Salem M, Abd El-Raheen TA, Aboraia NM (2018). Serum Copper and zinc levels in vitiligo patients. The Egyptian Journal of Hospital Medicine. 70(3): 364-370.

[23] Helmy MI, Gayyar EL, Hawas S et al. (2004). Role of oxidative stress in the pathogenesis of vitiligo. Journal of Pan-Arab League of Dermatologists. 15(3): 97-105. 
[24] Wasan TS, Al-Rubayee W (2011). Trace elements levels in serum and hair of patients with vitiligo and alopecia areata. Karbala Journal of Medicine. 4(2): 1117-1121.

[25] Wu Y, He N, Li JS et al. (2010). The zinc and copper levels in serum of 70 vitiligo patients from Guangxi Province. Chinese Journal of Dermatology Venereology. 24: 722.

[26] Shi DR, Pu XM, Ha LS (1993). A correlative study on serum copper and zinc in patients with vitiligo. Journal of Clinical Dermatology. 5: 241-243.

[27] Li YG, Zhou JG, Shao ZH (1988). Determination of the levels of copper and zinc in plasma of serum of some patients. Journal of Tianjin University of Commerce. 4: 24-30.

[28] Kang AJ, Su BS, Xu HQ (2002). Research on the melanocytes apoptosis in vitiligo caused by oxygen free radicals and microelement. Journal of Chinese Clinical Medicine. 3: 4-7. 